\title{
Sistema Web para estimativa de Evapotranspiração Potencial por diferentes métodos
}

\author{
Taison Anderson Bortolin' \\ Lucas Moraes dos Santos" \\ Adriano Gomes da Silvall' \\ Vania Elisabete Schneider ${ }^{\mathrm{IV}}$
}

Web System for estimation of Evapotranspiration Potential by different methods

\section{Resumo}

Um dos parâmetros do ciclo hidrológico que possui grande influência na gestão da água e na produção agrícola é a evapotranspiração. Encontrar formas de repor essa quantidade de água perdida, ao solo, é essencial, seja a partir das chuvas ou das técnicas de irrigação. Em geral, para determinar a evapotranspiração são utilizados métodos indiretos, destacando-se o de Penman-Monteith. No entanto, o método necessita de um grande número de variáveis sendo necessária a utilização de outros métodos indiretos, menos exigentes em relação aos dados requeridos. Devido a uma grande quantidade de métodos para serem comparados é interessante fazer o uso de ferramentas computacionais que permitam automatizar estes cálculos. Diante do problema, vislumbrou-se o desenvolvimento de uma aplicação web que efetua o cálculo da evapotranspiração de referência (ETo) de forma automatizada, a partir de diversos métodos matemáticos encontrados na literatura. O sistema dispõe ao usuário, resultados da ETo diária (mm/mês) para um determinado período de tempo, bem como a média diária $(\mathrm{mm} / \mathrm{mês})$, para o mês, e mensal ( $\mathrm{mm} / \mathrm{mês})$, para determinado mês durante o período de alguns anos, utilizando dados atualizados oriundos de estações meteorológicas operadas pelo INMET. A aplicação facilita o processo de cálculo minimizando o tempo de estimativa e contribuindo à análise dos resultados pelo usuário.

Palavras-chave: Evapotranspiração; Automatização; Web

\section{Abstract}

One of the parameters of the hydrological cycle that has great influence in water management and agricultural production is evapotranspiration. Finding ways to restore this amount of lost water to the ground is essential, whether from rain or irrigation techniques. In general, indirect methods are used to determine evapotranspiration, especially Penman-Monteith. However, the method requires a large number of variables, requiring the use of other indirect methods, less demanding in relation to the required data. Due to a great number of methods to be compared it is interesting to make use of computational tools that allow to automate these calculations. In the face of the problem, the development of a web application that calculates the reference evapotranspiration (ETo) in an automated way, from the several mathematical methods found in the literature, was glimpsed. The system provides the user with daily ETo results ( $\mathrm{mm} / \mathrm{month}$ ) for a given period of time, as well as the daily average ( $\mathrm{mm} / \mathrm{month}$ ), for the month, and monthly ( $\mathrm{mm} / \mathrm{month}$ ) for a given month during the period, using up-to-date data from INMET-operated weather stations. The application facilitates the calculation process minimizing the estimation time and contributing to the analysis of the results by the user.

Keywords: Evapotranspiration; Automation; Web

\footnotetext{
Universidade de Caxias do Sul, RS - Brasil. Email: tabortol@ucs.br

' Universidade de Caxias do Sul, RS - Brasil. Email: Imsantos7@ucs.br

I"' Universidade de Caxias do Sul, RS - Brasil. Email: agsilva11@ucs.br

ıv Universidade de Caxias do Sul, RS - Brasil.Email: veschnei@ucs.br
} 


\section{Introdução}

A evapotranspiração (ET) é um elemento importante do ciclo hidrológico e as possíveis tendências neste processo, entre outras coisas, podem influenciar a gestão da água e a produção agrícola (MACEK et al, 2018). A evapotranspiração pode ser considerada como um dos fatores mais importantes para indicar a mudança climática em bacias hidrográficas, uma vez que influencia tanto no escoamento superficial quanto no armazenamento de água na bacia (CANNAROZZO et al., 2006; LIU et al., 2008).

A ET é controlada e influenciada por diferentes fatores: temperatura média diária, velocidade dos ventos, umidade relativa, balanço de energia, demanda atmosférica, suprimento de água do solo às plantas e pelas características fisiológicas das plantas (MENDONÇA et al., 2003; PEREIRA et al., 2009, YANG et al., 2011, WANG et al., 2014). Em geral, é mais comum trabalhar com a evapotranspiração potencial (EPT), a qual pode ser definida como a taxa na qual a evapotranspiração (ET) ocorreria em uma grande área coberta completa e uniformemente com vegetação em crescimento, com acesso ilimitado a água, sem efeitos de advecção ou aquecimento (MCVICAR et al., 2012; MCMAHON et al., 2013).

Em função da dificuldade para realizar as medições diretas, a aplicação dos métodos indiretos tem se tornado uma ferramenta em potencial para a determinação da evapotranspiração (SULEIMAN e HOOGENBOOM, 2007, MOURA et al. 2013, PAIVA et al., 2016). Os métodos indiretos são utilizados para estimar a transferência de vapor d'água para a atmosfera em condições climáticas específicas, no entanto, poucos têm sido adaptados para situações climáticas distintas daquelas que lhes deram origem.

Existem diversos métodos para estimar a evapotranspiração, entre eles alguns baseados somente na temperatura, como o de Thornthwaite e outros baseados também na radiação solar, como o de Makkink e Jensen-Haise. Destaca-se ainda o método de Penman-Monteith, recomendado pela FAO (Food and Agriculture Organization of the United Nations) e utilizado para a calibração de outros métodos (ALLEN et al., 1998). No entanto, em alguns casos, o uso do método de Penman-Monteith é restrito pela falta de algumas variáveis de entrada, sendo necessária a utilização de outros métodos indiretos, menos exigentes em relação aos dados requeridos (GONÇALVES et al., 2009; PALARETTI et al., 2014; CRUZ, 2016; JÚNIOR et al., 2017). Diferentes autores recomendam que antes de aplicar um método para determinada região, é necessário verificar o desempenho deste e, se necessário, fazer calibrações com o objetivo de minimizar os erros de estimativa (LEITÃO et al., 2007; PEREIRA et al., 2009; PILAU et al., 2012).

Entretanto, considerando as dificuldades de processamento de dados e uma gama muito grande de métodos para serem comparados é interessante utilizar ferramentas computacionais que permitam automatizar estes cálculos contribuindo para uma análise mais eficaz da evapotranspiração em determinada região. Diferentes softwares podem ser encontrados na literatura, geralmente utilizando apenas o método de Penman-Montheit para estimativa de evapotranspiração (GOCIC e TRAKKOVIC, 2010; FEITOSA et al., 2014; GUO et al., 2016).

Com base no exposto, este trabalho tem por objetivo apresentar o desenvolvimento de uma aplicação web que visa automatizar o processo do cálculo de evapotranspiração, a partir de métodos matemáticos existentes na literatura, baseando-se em dados diários atualizados, advindos de diversas estações meteorológicas, com vistas a fornecer auxílio nas estimativas de evapotranspiração.

\section{Materiais e métodos}

O presente trabalho utilizou uma versão modificada da metodologia de desenvolvimento conhecida por Processo Unificado (PU), que sugere uma abordagem para modelagem e construção de software através de iterações curtas com resultados testáveis (LARMAN, 2007). Para modelar a aplicação, empregou-se o conceito de representação abstrata de processos que tem por objetivo descrever de forma abstrata os processos que compõem o sistema (SOMMERVILLE, 2007). A aplicação está baseada no paradigma cliente/servidor no qual um processo no host local, denominado cliente, solicita serviços a outro processo, normalmente localizado em um host remoto, denominado servidor (FOROUZAN, 2008). O servidor, por sua vez, retorna os serviços requisitados ao cliente, no momento que o usuário interage com a aplicação (SOMMERVILLE, 2007).

Para desenvolvimento da aplicação web foi empregado o padrão de arquitetura de software MVC (Model-View-Controller) que utiliza o paradigma de programação orientada a objetos (POO), um padrão de desenvolvimento que subdivide o software em componentes dispostos como modelo, visão e controlador, permitindo um melhor controle da qualidade do software, no que tange ao processamento dos dados e design de interface (HASAN e ISAAC, 2011).

Para o desenvolvimento programático do lado servidor (também conhecido como back-end) foi usada a linguagem de programação PHP (acrônimo recursivo para Hipertext Preprocessor), versão 7.0. O PHP caracteriza-se por ser uma linguagem de domínio específico (abrangendo o desenvolvimento web), cujo código é interpretado por um módulo PHP presente no lado servidor (PHP, 1995). No servidor, também é utilizada a linguagem de programação do pré-processador de texto Smarty Template Engine ${ }^{\text {nut }}$, para a geração e montagem dos templates dinâmicos (SMARTY TEMPLATE ENGINE, 2010).

No lado cliente (ou também front-end) as interfaces de usuário da aplicação foram desenvolvidas utilizando a linguagem de marcação HTML5 (do Inglês Hypertext Markup Language, versão 5), de estilos CSS3 (do Inglês Cascading Style Sheets, versão 3) e de scripting JavaScript (JS), empregando componentes do framework web, de código-fonte aberto, Bootstrap para desenvolvimento do design das páginas (BOOTSTRAP, 2013). O CSS e o JavaScript foram utilizados com o objetivo de estilizar e aprimorar a navegação do usuário no site. 
Algumas bibliotecas externas foram empregadas, dentre elas a OpenLayers para disponibilização do WebMapa interativo. A OpenLayers caracteriza-se por uma biblioteca modular javascript de alto desempenho e código aberto, com recursos para exibição e interação com mapas, além de permitir a manipulação de dados geoespaciais (OPENLAYERS, 2006). Outro fator que contribuiu à escolha da biblioteca foi devido a sua licença livre.

Na estruturação do banco de dados, foram desenvolvidos os Modelos Entidade - Relacionamento (MER) e Diagramas Entidade - Relacionamento (DER) da aplicação, por meio do plugin ER Master de construção de DERs, disponível para o ambiente integrado de desenvolvimento Eclipse (ERMASTER, 2013). Para armazenar os dados meteorológicos oriundos das estações, foi empregado o gerenciador de banco de dados PostgreSQL.

O trabalho, por utilizar do PU e suas Fases, foi desenvolvido em quatro etapas: concepção, elaboração, construção e transição. A etapa de concepção abrangeu o levantamento bibliográfico e pesquisa sobre sistemas que possuem funcionalidades semelhantes aos do projeto proposto. A elaboração consistiu na especificação do sistema, com base nos requisitos levantados, bem como na modelagem dos dados. Na construção foi realizada a implementação do sistema, ou seja, a conversão dos modelos ER para um banco de dados, o emprego de linguagens de programação usadas para definição de estruturas de dados, geração de código e o desenvolvimento das interfaces de funcionalidade do sistema, empregando linguagens de programação usadas no desenvolvimento de aplicações web. Por fim, na transição, houve a disponibilização do sistema ao usuário final e avaliação dos resultados obtidos (SCHACH, 2010).

\section{Modelagem dos dados}

Modelos de dados são ferramentas que permitem demonstrar como serão construídas as estruturas de dados que darão suporte aos processos do sistema, como esses dados estarão organizados e quais relacionamentos pretende-se estabelecer entre eles (DEBASTIANI, 2015). Para obter os dados meteorológicos optou-se por utilizar o Sistema de Monitoramento Agrometeorológico (Agritempo), que tem como mantenedores a Embrapa Informática Agropecuária e o Centro de Pesquisas Meteorológicas e Climáticas Aplicadas à Agricultura, da Universidade Estadual de Campinas (Unicamp) (AGRITEMPO, 2002). A escolha do Agritempo foi motivada pelo fato do sistema conter mais parâmetros meteorológicos.

Cada conjunto de dados é formado por parâmetros meteorológicos medidos diariamente nas estações meteorológicas e assim discriminados: temperatura, umidade relativa do ar, precipitação, radiação solar, pressão atmosférica, direção e velocidade do vento, pontos de orvalho e disponibilidade de água do solo. Na conversão do DER em Linguagem de definição de dados (DDL - do inglês, Data Definition Language), as entidades passaram a ser representadas por tabelas e os atributos, por colunas. Estes dados foram diferenciados pelo identificador da estação correspondente em banco de dados. No caso de não existirem informações armazenadas em banco, para o período consultado pelo usuário, uma nova consulta ao Agritempo é realizada usando a data do último registro de dados armazenado em banco e a data solicitada como chave de busca. Assim o conteúdo em banco de dados estará atualizado.

\section{Modelagem da aplicação}

Na modelagem da aplicação foram desenvolvidas as estruturas base do padrão MVC (responsáveis pelo armazenamento e consulta dos dados), o controle das interações usuário-sistema, a renderização da página inicial e o núcleo de funcionalidades base do sistema. A partir destas estruturas, os dados meteorológicos puderam ser processados e armazenados em banco de dados, estando disponíveis ao usuário para posterior análise. Também foi realizado o desenvolvimento programático dos métodos matemáticos, empregados na estimativa de ETo.

Após a aplicação dos métodos matemáticos sobre os dados de estações meteorológicas consultados, são obtidos os resultados de ETo. Com vistas a possibilitar uma melhor exibição dos pontos das estações meteorológicas e interação com o mapa, empregou-se um WebMapa. Por fim, desenvolveu-se o gerenciamento de usuários, responsável pelo cadastro de novos usuários ao sistema e controle de acesso a aplicação através de uma política de níveis de usuários.

\section{Construção}

Após a modelagem dos dados, desenvolveu-se a lógica responsável pelo controle das interações entre usuário e sistema, bem como o desenvolvimento programático dos métodos matemáticos. Conforme o uso do desenvolvimento iterativo proveniente do $\mathrm{PU}$, optou-se por armazenar previamente uma faixa de dados meteorológicos em banco de dados e delegar à Controladora o gerenciamento da consulta ao Agritempo. Essa abordagem foi estabelecida após as consultas ao servidor externo tornarem-se demasiadamente lentas para longos períodos de tempo.

Inicialmente, a Controladora cria uma instância do Modelo, responsável pela conexão com o banco de dados, e do Visualizador que se encarrega de construir e apresentar a página inicial. A página inicial é composta por um formulário onde o usuário deverá inserir as informações necessárias de acesso ao sistema. Os elementos da página inicial são processados pelo componente Smarty. Este componente é utilizado no sistema por meio do Visualizador, que coleta os dados e ações em nível de execução do código PHP e, antes do retorno ao cliente da página .html, processa o .tpl e gera o arquivo .html. A Controladora, por sua vez, envia uma solicitação ao Modelo, contendo as informações do usuário encapsuladas junto à requisição ao servidor.

Cabe a Controladora também o gerenciamento do processo de consulta aos dados meteorológicos das estações, realizada por meio de um método POST. Cada consulta contém as informações do formulário (código identificador 
Tabela 1 - Métodos para cálculo de evapotranspiração potencial

\begin{tabular}{|c|c|c|}
\hline Método & Equação & Variáveis \\
\hline $\begin{array}{c}\text { Thornthwaite } \\
\text { (1948) }\end{array}$ & $\begin{array}{l}\text { ETo }=16 *\left(10 * \frac{T_{i}}{I}\right)^{a}, 0{ }^{\circ} \mathrm{C} \leq T_{i} \leq 26^{\circ} \mathrm{C} \\
\text { ETo }=-415,85+32,24 T_{i}-0,43 T_{i}^{2}, T_{i}>26^{\circ} \mathrm{C} \\
=\sum_{i=1}^{12}\left(0,2 T_{i}\right)^{1,514}, T_{i} \geq 0{ }^{\circ} \mathrm{C} \\
a=6,75 * 10^{-7} I^{3}-7,71 * 10^{-5} I^{2}+1,7912 * 10^{-2} I+0,49239 \\
C=\frac{N}{12} * \frac{1}{30} \\
N=\frac{2}{12} * \omega_{s}\end{array}$ & $\begin{array}{l}\text { • ETo = } \\
\text { Evapotranspiração } \\
\text { de referência (mm } \\
\text { mês }{ }^{-1} \text { ); } \\
\text { - } \mathrm{T}_{\mathrm{i}}=\text { Temperatura do } \\
\text { ar média mensal }\left({ }^{(} \mathrm{C}\right) ; \\
\text { • I = Índice térmico } \\
\text { imposto pelo regime } \\
\text { climático local; } \\
\text { • i = Representa o mês } \\
\text { do ano (i=1, janeiro; .... } \\
\mathrm{i}=12 \text {, dezembro); } \\
\text { - } \mathrm{N}=\text { Fotoperíodo (h) } \\
\text { de determinado dia; }\end{array}$ \\
\hline $\begin{array}{c}\text { Thornthwaite } \\
\text { (1948) - } \\
\text { Modificado }\end{array}$ & $\begin{array}{l}T_{e f}=K *\left(T_{i}+A\right) \\
T=\left(\frac{T_{\max }+T_{\min }}{2}\right) \\
A=T_{\max }-T_{\min } \\
T_{e f}=0,5 k *\left(3 T_{\max }-T_{\min }\right)\end{array}$ & $\begin{array}{l}\text { - } \mathrm{A}=\text { Amplitude } \\
\text { térmica diária; } \\
\text { - } \mathrm{T}_{\max }=\text { Temperatura } \\
\text { máxima }\left({ }^{\circ} \mathrm{C}\right) \text {; } \\
\text { - } \mathrm{T}_{\min }=\text { Temperatura } \\
\text { mínima }\left({ }^{\circ} \mathrm{C}\right) \\
\cdot \mathrm{k}=0,72 \text { e } \mathrm{k}=0,69\end{array}$ \\
\hline $\begin{array}{c}\text { Hargreaves - } \\
\text { Samani }\end{array}$ & $\begin{array}{l}E T o_{(H S)}=0,0135 * k t * R a *\left(T_{\max }-T_{\min }\right)^{0,5} *\left(T_{\text {med }}+17,8\right) \\
E T o_{(H S)}=0,0023 * R_{T} * \sqrt{T_{\max }-T_{\min }} *(T+17,8)\end{array}$ & 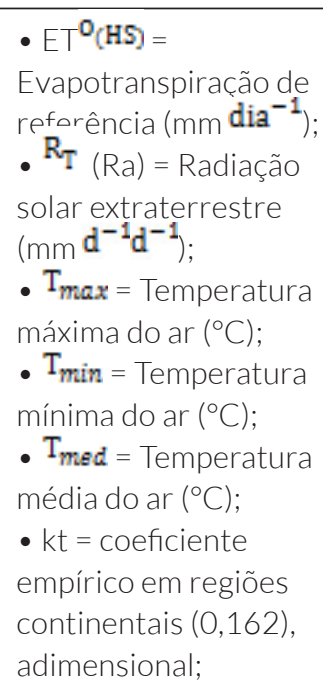 \\
\hline
\end{tabular}

Continuação... 
Tabela 1 - Métodos para cálculo de evapotranspiração potencial

\begin{tabular}{|c|c|c|}
\hline Método & Equação & Variáveis \\
\hline $\begin{array}{l}\text { Blaney - } \\
\text { Criddle }\end{array}$ & $\begin{array}{l}E T o_{(B C)}=a+b * f \\
f=p *(0,46 * T+8,13) \\
a=0,0043 * U R_{\min }-\frac{n}{N}-1,41 \\
b=a_{0}+a_{1} * U R_{\text {min }}+a_{2} * \frac{n}{N}+a_{3} * u_{2}+a_{4} * U R_{\min } * \frac{n}{N}+a_{5} * U R_{\text {min }} * u_{2}\end{array}$ & 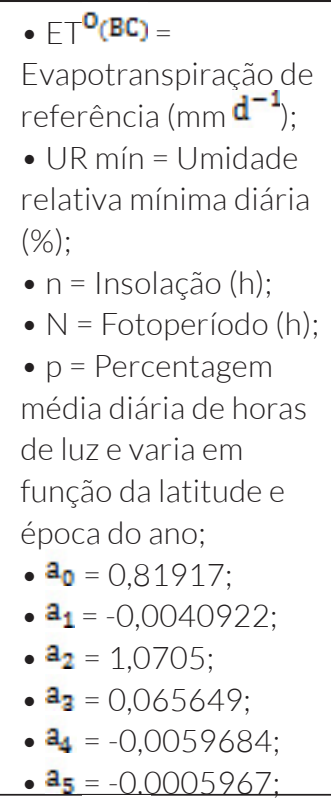 \\
\hline Camargo & $E T o_{(C A)}=R_{T} * T * K_{f} * N D$ & 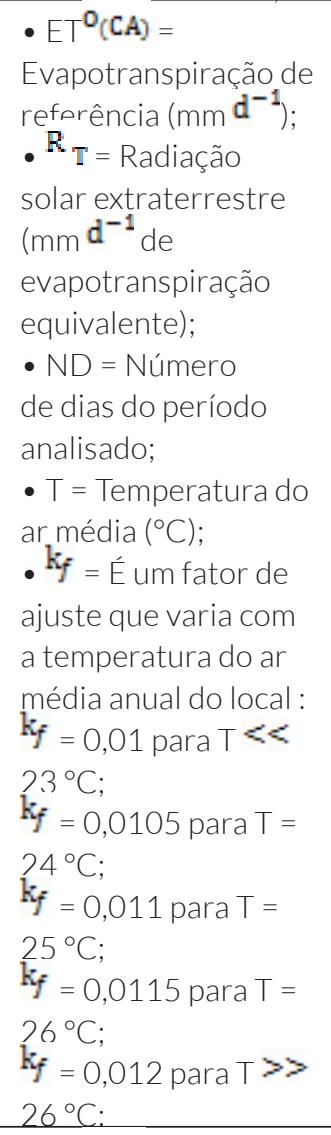 \\
\hline Jensen - Haise & $\begin{array}{l}E T o_{(J H)}=R_{s} *(0,0252 * T+0,078) \\
E T o_{(J H)}=R_{s} *\left(0,025 * T_{m e d}+0,08\right)\end{array}$ & $\begin{array}{l}\text { - } E T^{0}(\mathrm{JH})= \\
\text { Evapotranspiração de } \\
\text { referência }\left(\mathrm{mm} \mathrm{d}^{-1}\right) ; \\
\text { - } \mathrm{R}_{s=\text { Radiação solar }} \\
\text { global convertida em } \\
\text { unidades de água } \\
\left.\text { evaporada (mm dia }{ }^{-1}\right) \text {; } \\
\text { - } \mathrm{T}_{\text {med }}=\text { Temperatura } \\
\text { diária média do } \operatorname{ar}\left({ }^{\circ} \mathrm{C}\right) \text { : }\end{array}$ \\
\hline
\end{tabular}


Tabela 1 - Métodos para cálculo de evapotranspiração potencial

\begin{tabular}{|c|c|c|}
\hline Método & Equação & Variáveis \\
\hline Makkink 7 & $E T o_{(M K)}=0,61 * W * R_{T}-0,12$ & $\begin{array}{l}\text { - } \mathrm{ET}^{0}(\mathrm{MK})= \\
\text { Evapotranspiração de } \\
\text { referência }\left(\mathrm{mm}^{-1} \mathrm{~d}^{-1}\right) ; \\
\text { - W = Fator de } \\
\text { ponderação } \\
{ }^{*} \mathrm{O} \text { fator W varia em } \\
\text { função da temperatura } \\
\text { do bulbo úmido. }\end{array}$ \\
\hline $\begin{array}{l}\text { Benavides - } \\
\text { Lopez (BL) } 8\end{array}$ & $E T o_{(B L)}=1,21 * 10 *\left(\frac{7,45 * T_{m e d}}{234,7+T_{m e d}}\right) *(1-0,01 * U R)+0,21 * T_{m e d}-2,30$ & 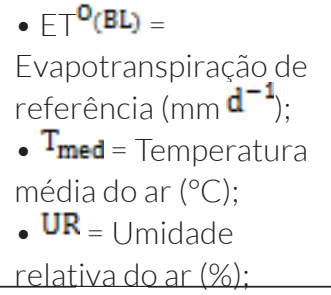 \\
\hline Hamon (HA) 9 & $E T o_{(H A)}=0,55 *\left(\frac{N}{12}\right)^{2} *\left(\frac{4,93 e^{\left(0,062 T_{m e d}\right)}}{100}\right) * 25,4$ & $\begin{array}{l}\text { - } \mathrm{ET}^{0}(\mathrm{HA})= \\
\text { Evapotranspiração de } \\
\text { referência }\left(\mathrm{mm}^{-1} \mathrm{~d}^{-1}\right) \\
\text { - } \mathrm{N}=\text { Número de } \\
\text { horas de brilho } \\
\text { solar possíveis } \\
\text { (fotoberíodo); } \\
\text { - } \mathrm{T}_{\text {med }}=\text { Temperatura } \\
\text { média do ar }\left({ }^{\circ} \mathrm{C}\right) \text {; }\end{array}$ \\
\hline $\begin{array}{l}\text { Priestley \& } \\
\text { Taylor (PT) } 10\end{array}$ & $E T o_{(P T)}=\frac{\alpha \frac{(\Delta+\gamma)}{\gamma} *\left(R_{n}-G\right)}{\delta}$ & 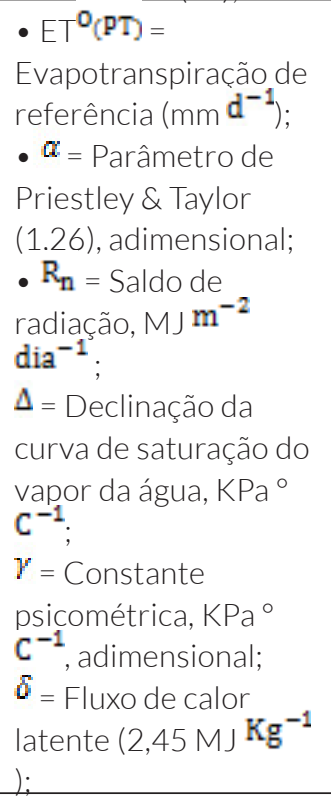 \\
\hline
\end{tabular}

Continuação... 
Tabela 1 - Métodos para cálculo de evapotranspiração potencial

\begin{tabular}{|c|c|c|}
\hline Método & Equação & Variáveis \\
\hline Turc (TU) 11 & $E T o_{(T U)}=a_{t} 0,013 *\left(\frac{T_{m e d}}{T_{m e d}+15}\right) *\left(\frac{R_{s}}{\frac{0,0238846}{\gamma}+50}\right)$ & 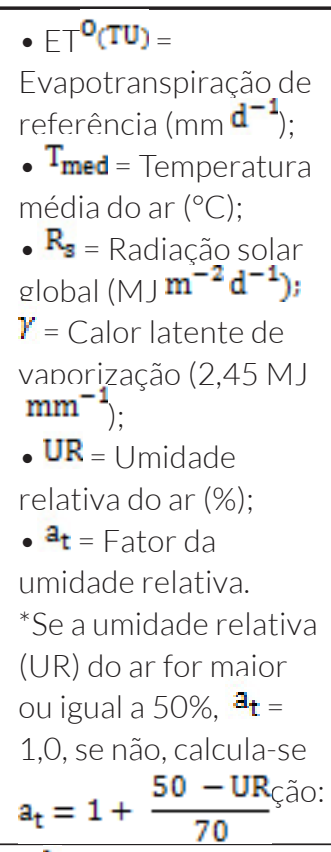 \\
\hline Linacre 12 & $\begin{array}{l}E T o=\frac{500 *\left(\frac{T_{m e d}+0,006 * h}{100-\varphi}\right)+15 *\left(T_{m e d}-T_{p o}\right)}{80-T_{m e d}} \\
E T o=\frac{J *\left(\frac{T_{a}-0,006 * z}{100-\emptyset}\right)+15 *\left(T_{a}-T_{o}\right)}{80-T_{a}} \\
T_{p o}=\frac{237,3 * \log \left(e_{a}\right)-156,8}{8,16-\log \left(e_{a}\right)}\end{array}$ & 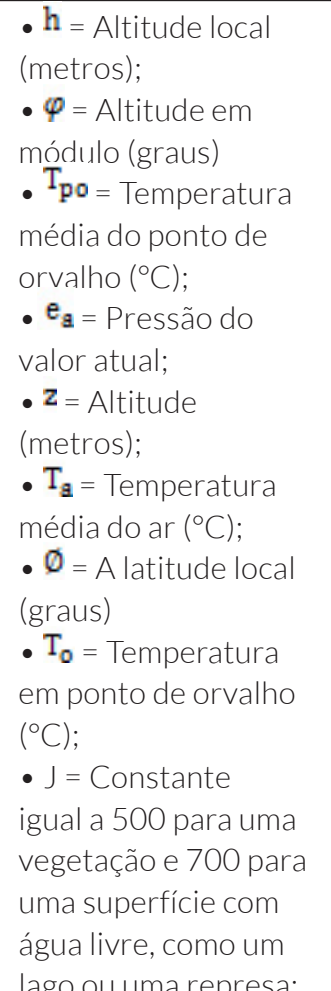 \\
\hline Kharrufa & $E T o=0,34 * p * T_{m e d}^{1,3}$ & 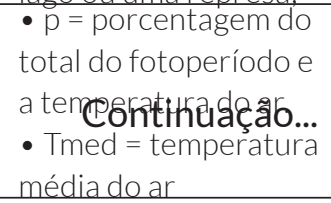 \\
\hline
\end{tabular}

Continuação... 
Tabela 1 - Métodos para cálculo de evapotranspiração potencial

\begin{tabular}{|c|c|c|}
\hline Método & Equação & Variáveis \\
\hline $\begin{array}{l}\text { Penman- } \\
\text { Monteith } 14\end{array}$ & $E T o=\frac{0,408 \Delta\left(R_{n}-G\right)+\gamma\left(\frac{900}{T+273}\right) \mu_{2}\left(e_{s}-e_{a}\right)}{\Delta+\gamma\left(1+0,34 \mu_{2}\right)}$ & 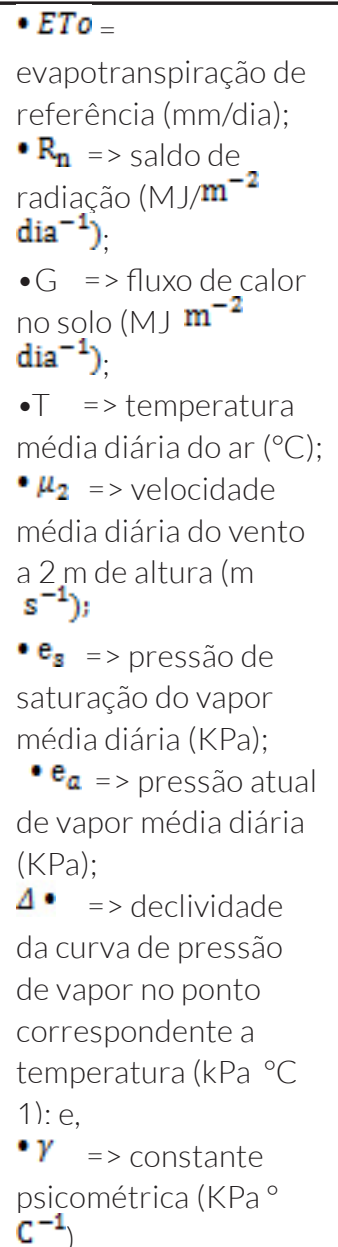 \\
\hline
\end{tabular}

Figura 1 - Diagrama de sequência para o método de Hargreaves e Samani (1985)

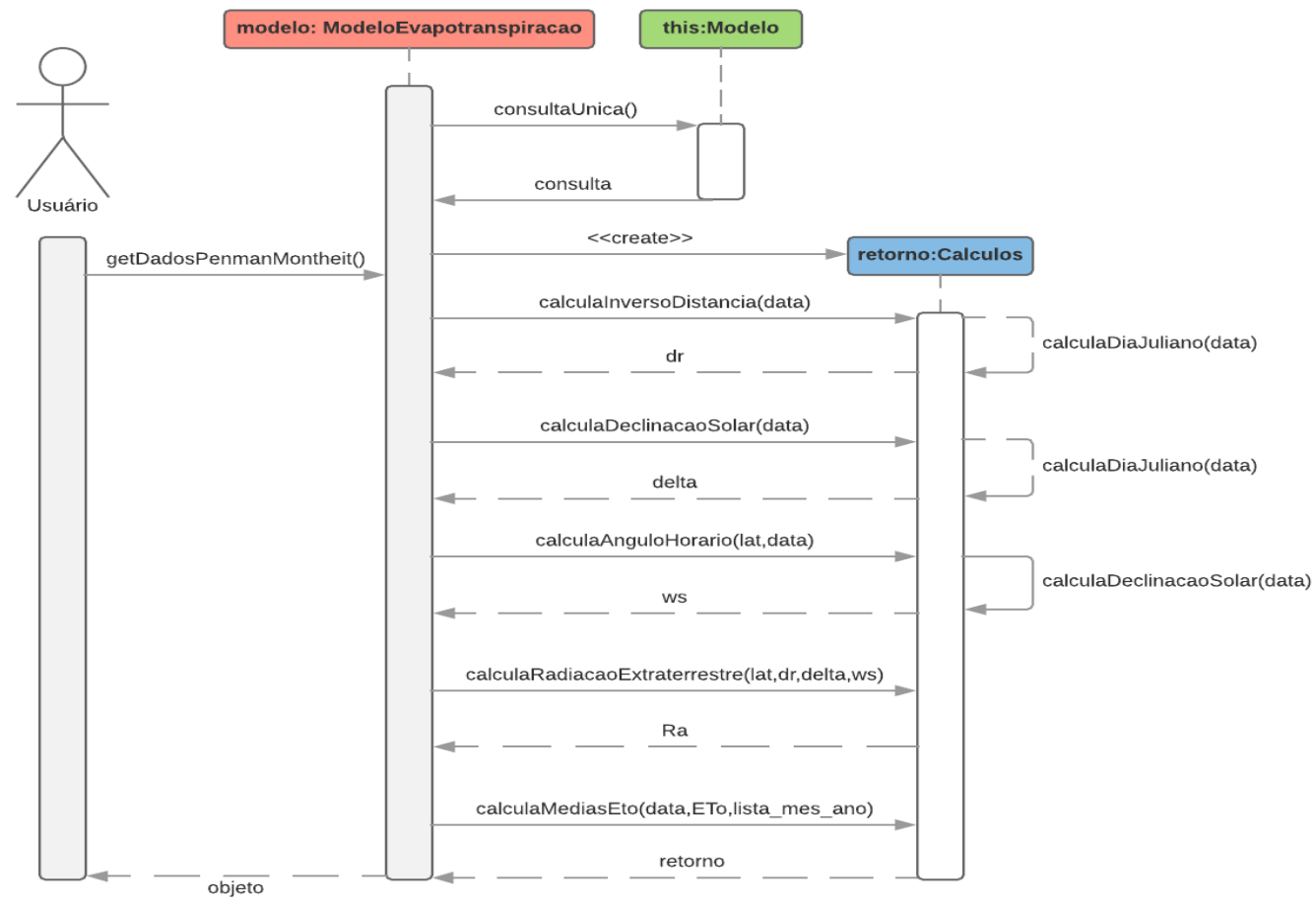

Fonte: Elaborado pelo autor 
da estação e período da pesquisa) encapsuladas junto à requisição ao servidor externo. Com o retorno dos dados meteorológicos, a Controladora envia-os ao Visualizador que os apresenta ao usuário. Em seguida, o usuário deverá optar por um dos métodos matemáticos para estimar a ETo sobre os dados consultados. Atualmente, existe um total de 14 métodos matemáticos implementados para estimativa da ETo os quais são apresentados na Tabela 1.

A Controladora envia uma instrução ao Modelo indicando quais métodos matemáticos devem ser executados. Cada método foi implementado programaticamente no Modelo que também se encarrega da consulta dos dados meteorológicos em banco de dados. Estes dados correspondem aos parâmetros meteorológicos específicos à execução de método selecionado. Sobre cada conjunto de dados estima-se a ETo e, posteriormente, os resultados obtidos são enviados à Controladora. Esta, por sua vez, envia-os ao Visualizador, para que este exiba as informações resultantes ao usuário da aplicação.

Com vistas a obter parâmetros intermediários para estimativa da ETo, os quais não estão presentes no conjunto de dados advindos das estações meteorológicas, foram implementados métodos auxiliares para obtenção desses parâmetros. Dessa forma, reduz-se o problema da redundância de código pertinente a diversos métodos utilizando as mesmas variáveis, criando classes com domínios mais específicos. O diagrama de sequência de execução para o método matemático de Hargreaves-Samani (1985) é apresentado na Figura 1.

A gerência de usuários foi implementada com o intuito de manter o controle de quem acessa o sistema, bem como informações sobre qual instituição está utilizando a aplicação. O gerenciamento de usuários opera baseado em uma política de níveis de usuário onde o nível de usuário logado é o nível com menor acesso e o de administrador maior. O maior nível herda as funções do menor. $\mathrm{O}$ administrador tem a função de manter o controle dos usuários, podendo restringir o acesso, excluir contas, se necessário, etc. O usuário logado poderá consultar os dados meteorológicos, além de aplicar os métodos matemáticos sobre os dados consultados, obtendo as estimativas de ETo para o período consultado. A leitura das coordenadas do banco de dados e inserção dos pontos no mapa são realizadas utilizando javascript.

\section{Resultados e discussões}

Para acessar a aplicação, é necessária a realização de um cadastro prévio que conterá algumas informações tais como nome, usuário, endereço eletrônico, senha e instituição, necessárias ao registro do usuário a aplicação. $\mathrm{O}$ acesso a aplicação pode ser realizado através do link https:// siambiental.ucs.br/intermediador/. As informações para cadastro de usuário podem ser visualizadas na Figura 2.

A página de acesso à aplicação pode ser visualizada na Figura 3, onde o usuário deverá inserir suas informações de usuário e senha caso deseje utilizar do sistema.

Os dados das estações meteorológicas podem ser con- sultados através de um WebMapa, ilustrado na Figura 4, onde as estações são representadas por meio de pontos.

Ao selecionar uma estação meteorológica (Figura 5), o usuário deverá inserir o período que deseja efetuar a pesquisa. Caso não existam dados armazenados no banco de dados para determinado período, uma consulta ao servidor remoto com parâmetros de requisição é realizada e o retorno é utilizado para atualizar os dados da estação meteorológica em banco.

Os dados meteorológicos, resultantes da consulta, são apresentados ao usuário, conforme ilustra a Figura 6. O usuário tem a opção de baixá-los no formato .csv, para posterior análise.

A partir da consulta aos dados meteorológicos, o usuário deverá escolher, dentre os métodos matemáticos (Tabela 1), quais pretende executar para estimar a ETo. Caso o usuário selecione mais de um método matemático para estimar a ETo, o sistema insere os resultados em um arquivo .csv e este é disponibilizado para o usuário. No entanto, se apenas um método é selecionado, o sistema disponibiliza os resultados em uma tabela, conforme ilustrado na Figura 7.

Após aplicar os métodos matemáticos sobre os dados consultados, são obtidos os resultados para a ETo diária, ETo média diária mensal em milímetros por dia ( $\mathrm{mm} / \mathrm{dia})$, e a ETo média mensal em milímetros por mês ( $\mathrm{mm} / \mathrm{mês})$. A título de exemplo, a Figura 6 apresenta os resultados do cálculo da ETo diária, média diária mensal e média mensal, a partir do método de Penman-Monteith-FAO, para um período compreendido entre 01/01/2016 a 02/12/2018 tendo por base os dados da estação meteorológica do município de Porto Alegre - RS (dados disponíveis de 01/01/1965 a 03/12/2018).

$\mathrm{O}$ administrador poderá gerenciar os usuários através de uma tabela (Figura 8) contendo as informações cadastradas.

Para controlar o acesso de determinado usuário a aplicação, o administrador poderá alterar a situação deste, a partir da tela de edição de informações do usuário, apresentada na Figura 9.

A possibilidade de aplicação de diversos modelos matemáticos para cálculo de evapotranspiração utilizando dados meteorológicos e sua disponibilização como um sistema web auxilia os pesquisadores evitando a necessidade de implementação de novos algoritmos para resolução de um método em específico. Assim, viabiliza a alocação do tempo anteriormente direcionado ao desenvolvimento de ferramentas computacionais específicas para diferentes métodos, para análises sobre os resultados fornecidos pelo sistema.

$\mathrm{O}$ arquivo resultante do cálculo (arquivo em formato .csv) gerado com a utilização de mais de um método para estimativa de ETP permite, além disso, uma análise comparativa de diversos modelos, cuja relação e análise dos resultados gerados fica a critério do usuário. A disponibilização dos dados brutos e compilados em planilha eletrônica é um modo de aprimorar a aplicação de modelos desenvolvida, sendo pertinente a diversos trabalhos já desenvolvidos, nos quais foram aplicados múltiplos métodos de comparação (GONÇALVES et 
Figura 2 - Formulário de cadastro ao sistema

Intermediador

\section{Cadastrar usuário}

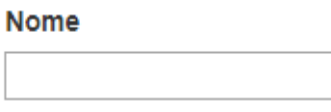

Senha

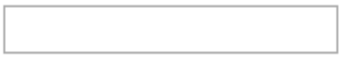

\section{Usuário}

Email

Instituição

Limpar Enviar

Fonte : Elaborado pelo autor.

Figura 3 - Formulário de acesso ao sistema

Intermediador

\section{Entrar no sistema}

Usuário $\square$ Senha $\square$ Login

Fonte: Elaborado pelo autor.

Figura 4 - WebMapa dispondo as estações meteorológicas do Estado do Rio Grande do Sul

Intermediador

Estimativa de ETo \& Separação de Escoamento - Selecione uma opção de ponto no menu abaixo

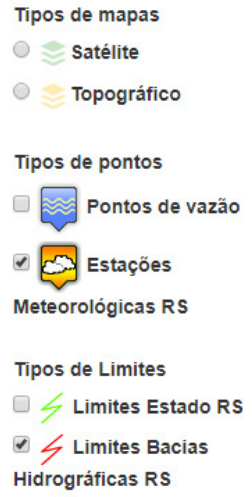

Fonte: Elaborado pelo autor

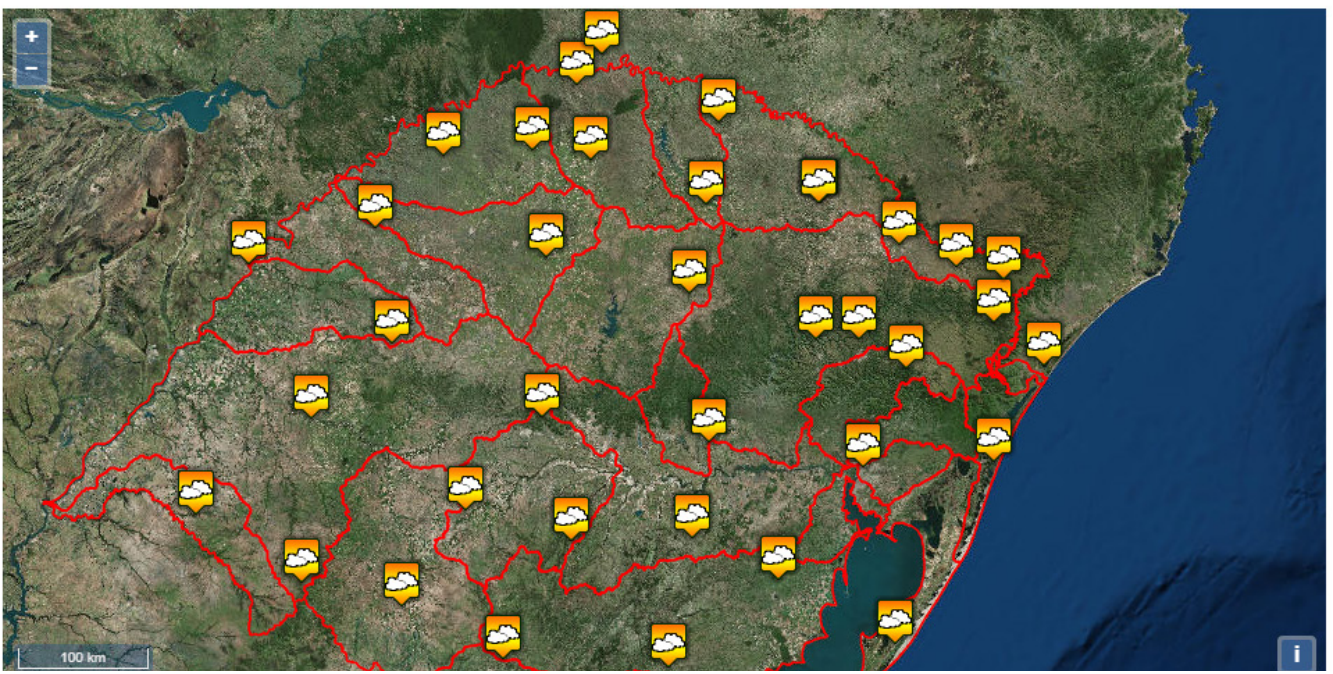


Figura 5 - Seleção do ponto correspondente a estação meteorológica do município de Porto Alegre - RS - período de análise: 01/01/2016 a 02/12/2018.

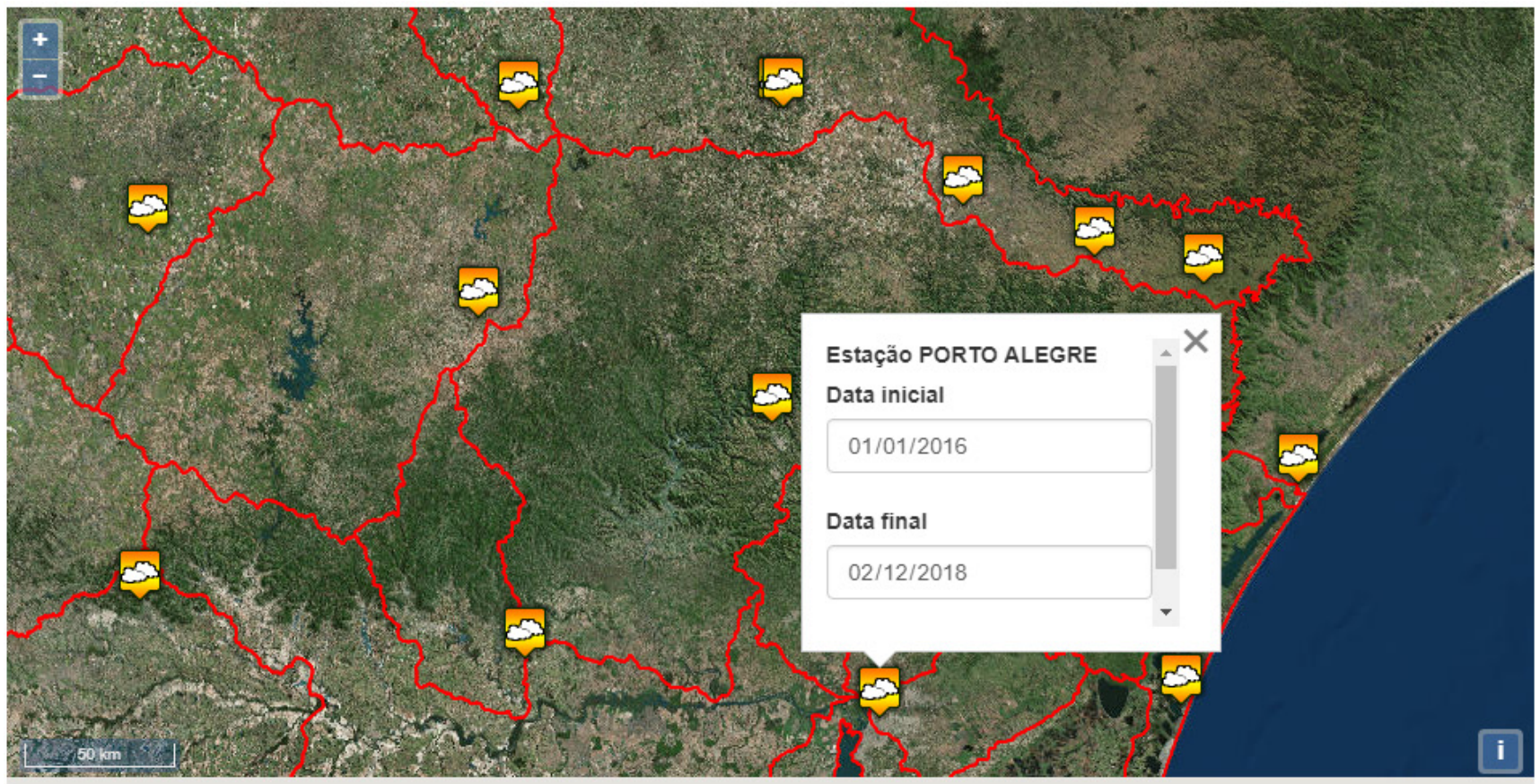

Fonte: Elaborado pelo autor

Figura 6 - Dados meteorológicos consultados para a estação meteorológica do município de Porto Alegre - RS - período de análise: 01/01/2016 a 02/12/2018.

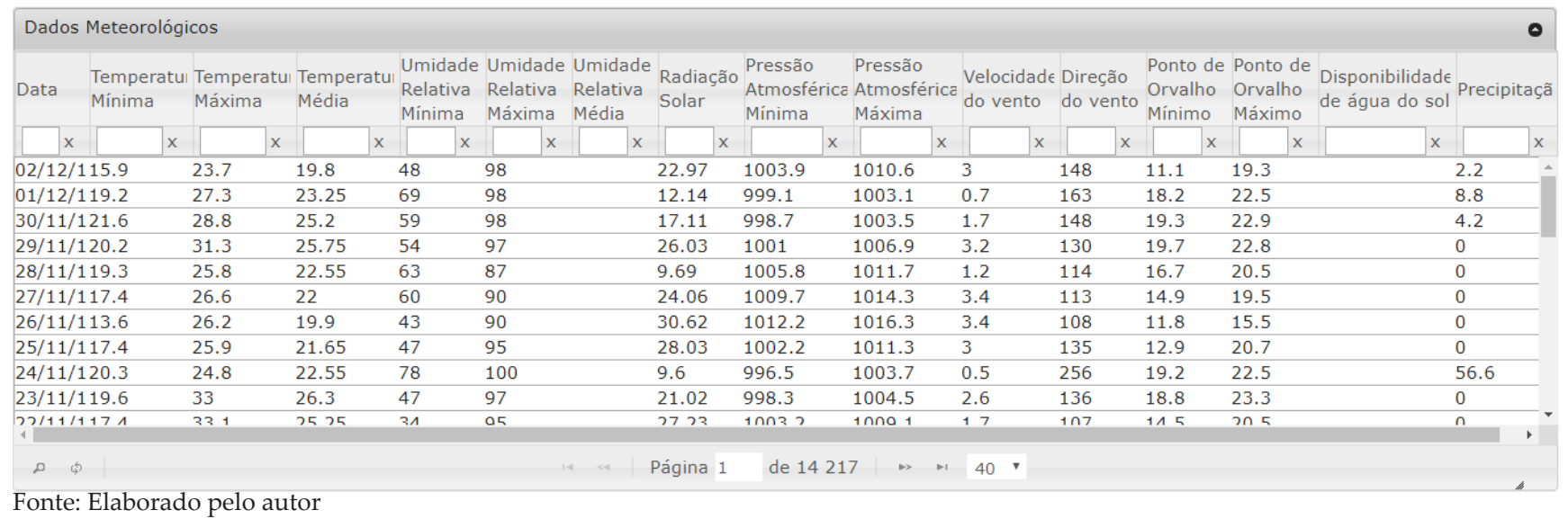

Fonte: Elaborado pelo autor

Figura 7 - Resultados de ETo para a estação meteorológica do município de Porto Alegre - RS - período de análise: $01 / 01 / 2016$ a $02 / 12 / 2018$

\begin{tabular}{|l|l|l|l|l|l|}
\hline \multicolumn{1}{|c|}{ Periodo analisado } & \multicolumn{1}{|c|}{ Evapotranspiraçāo (mm/dia) } & \multicolumn{1}{|c|}{ Mès/Ano } & Média diária mensal (mm/dia) & \multicolumn{1}{|c|}{ Meses } & \multicolumn{1}{c|}{ Média mensal (mm/mês) } \\
\hline $02 / 12 / 18$ & 5.55 & Dezembro/2018 & 5.26 & Dezembro & 127.86 \\
\hline $01 / 12 / 18$ & 4.98 & Novembro/2018 & 6.2 & Novembro & 162.84 \\
\hline $30 / 11 / 18$ & 5.47 & Outubro/2018 & 5.13 & Outubro & 146.09 \\
\hline $29 / 11 / 18$ & 6.56 & Setembro/2018 & 3.99 & Setembro & 116.66 \\
\hline $28 / 11 / 18$ & 5.36 & Agosto/2018 & 3.18 & Agosto & 78.05 \\
\hline $27 / 11 / 18$ & 5.61 & Julho/2018 & 2.52 & Julho & 75.18 \\
\hline $26 / 11 / 18$ & 6.39 & Junho/2018 & 2.24 & Junho & 60.77 \\
\hline
\end{tabular}

Fonte: Elaborado pelo autor 
Figura 8 - Tabela de controle dos usuários cadastrados no sistema

\begin{tabular}{|c|c|c|c|c|c|c|c|}
\hline \multicolumn{7}{|l|}{ Usuários } & \multirow[t]{2}{*}{ Q } \\
\hline Id Usuário & Nome & & Email & Instituição & & Situação & \\
\hline $\mathrm{x}$ & & $\mathrm{x}$ & & $x$ & $x$ & & $x$ \\
\hline 1 & Usuário 1 & & user1@gmail.com & A & & Ativo & \\
\hline 2 & Usuário 2 & & user2@gmail.com & B & & Ativo & \\
\hline 3 & Usuário 3 & & user3@gmail.com & C & & Ativo & \\
\hline
\end{tabular}

Fonte: Elaborado pelo autor

Figura 9 - Formulário para edição de informações de usuários

Intermediador

\section{Editar usuários}

Nome
Usuário 1

Limpar Enviar

Fonte: Elaborado pelo autor

Usuário
$\begin{aligned} & \text { User_1 } \\ & \text { Instituição } \\ & \text { A }\end{aligned}$

Email

user_1@gmail.com

Situação

\begin{tabular}{|l|}
\hline Ativo \\
\hline Ativo \\
\hline Desativo
\end{tabular}

al., 2009; OLIVEIRA et al., 2010; JÚNIOR et al., 2012; CHAGAS et al., 2013).

Entretanto, os usuários devem estar cientes das suposições e limitações completas antes de usar qualquer modelo ET nesta aplicação web. Quase todo modelo de ET contém suposições relacionadas às condições climáticas específicas sob as quais os modelos se aplicam. Por exemplo, alguns modelos assumem que os subprocessos relacionados a ET são insignificantes, enquanto outros modelos são apenas calibrados para o clima de uma região específica (ROSENBERRY et al., 2007; TABARI et al., 2013; GUO et al., 2016).

\section{Conclusão}

O sistema proporcionou uma forma automatizada de efetuar o cálculo de ETo, a partir de diversos métodos matemáticos e para longos períodos de tempo. Devido ao nível de complexidade matemática de alguns métodos, o processo de estimativa de evapotranspiração tornar-se-ia inviável, em especial, quando os períodos de análise compreendem muitos anos ou ainda, quando não estão disponíveis alguns parâmetros. Como salienta Oliveira et al. (2010), devido a característica empírica presente em alguns métodos usados no cálculo de ETo, a obtenção dos resultados, por vezes, fica comprometida devido a indisponibilidade de determinados parâmetros meteorológicos a uma região. Com vistas a contornar esse problema, a aplicação dispõe de um número considerável de técnicas alternativas que se utilizam de diferentes parâmetros para calcular a ETo, tendo o usuário a opção de escolher qual técnica é mais adequada para seu estudo. Além disso, o sistema possui a opção de executar mais de um método concomitantemente, proporcionando uma forma prática ao usuário que deseja realizar análises.

O emprego do WebMapa possibilitou ao usuário maior facilidade no processo de consulta dos dados meteorológicos. A partir de uma representação visual dos pontos climatológicos disponíveis, o usuário pode selecionar a estação meteorológica desejada e realizar a consulta pretendida. No tocante a gerência dos usuários, o sistema provê uma forma hierárquica de acesso aos recursos da aplicação. O administrador poderá excluir 
usuários de forma prática, bem como editar algumas informações cadastradas. O usuário, por sua vez, poderá efetuar diversas análises sobre os dados consultados, de acordo com sua necessidade.

A validação dos resultados vem sendo constantemente realizada, bem como a correção de possíveis erros. Além disso, vem sendo desenvolvido o estudo de técnicas atuais que proporcionem as melhorias à aplicação, tanto na usabilidade, quanto na disponibilização de novas funcionalidades, dentre elas a elaboração de gráficos e análises estatísticas e de desempenho dos métodos.

\section{Referências}

ALLEN, R.G. et al. Crop evapotranspiration. Guidelines for computing crop water requirements. Rome: FAO, 1998. 299p.

AGRITEMPO. Agritempo: Sistema de Monitoramento Agrometeorológico. Disponível em: http://www. agritempo.gov.br . Acesso em: fev. 2019.

BOOTSTRAP, T. Twitter bootstrap. 2013. Disponível em: http://getbootstrap.com.

BORGES JÚNIOR, João C. F. et al. Métodos de estimativa da evapotranspiração de referência diária para a microrregião de Garanhuns, PE. Revista Brasileira de Engenharia Agrícola e Ambiental, Campina Grande, v. 16, n. 4, p.380-390, jan. 2012.

CANNAROZZO, L.V. Noto, F. Viola. Spatial distribution of rainfall trends in Sicily (1921-2000) Phys. Chem. Earth, 31 (18) (2006), pp. 1201-1211

DEBASTIANi, Carlos Alberto. Definindo Escopo em Projetos de Software. São Paulo: Novatec, 2015. 144 p.

ECLIPSE FOUNDATION. Eclipse Process Framework. Disponível em: https:/www.eclipse.org/. Acesso em: 03 de Fev. de 2019.

EMBRAPA. Documentos 263 - Evapotranspiração-Uma Revisão sobre os Métodos Empíricos. Empresa Brasileira de Pesquisa Agropecuária Embrapa Arroz e Feijão Ministério da Agricultura, Pecuária e Abastecimento, 2010.

ERMASTER. What is ERMaster ?. Disponível em: http://ermaster.sourceforge.net/. Acesso em: 03 de Fev. de 2019.

FANAYA JÚNIOR, Eder Duarte et al. Métodos empíricos para estimativa da evapotranspiração de referência para Aquidauana, MS. Irriga, Botucatu, Sp, v. 17, n. 4, p.418434, 02 out. 2012.

FEITOSA, D. G. et al. Artigo técnico software Smai 2.0 para estimativa da evapotranspiração de referência diária e horária 1. [s. 1.], [s.d.]. Disponível em: http://clima.feis. unesp.br/smai

FOROUZAN B. A. Comunicação de Dados e Redes de Computadores. 4. ed. São Paulo: McGraw-Hill, 2008.

GONÇALVES, Fabrício Mota et al. Comparação de métodos da estimativa da evapotranspiração de referência para o município de Sobral-CE. Revista Brasileira de Agricultura Irrigada, Fortaleza, Ce, p.1-8, 12 ago. 2009.

GOCIC, Milan; TRAJKOVIC, Slavisa. Software for estimating reference evapotranspiration using limited weather data. Computers and Electronics in Agriculture, [s. 1.], v. 71, n. 2, p. 158-162, 2010.

GUO, D., S. WESTRA, AND H. R. MAIER, An R package for modelling actual, potential and reference evapotranspiration, Environ. Model. Software, 78, 216-224, 2016.

HASAN, S.s.; ISAAC, R.k.. An integrated approach of MAS-CommonKADS, Model-View-Controller and web application optimization strategies for web-based expert system development. Expert Systems With Applications, [s.l.], v. 38, n. 1, p.417-428, jan. 2011. Elsevier BV.

JÚNIOR, A.S.A. Avaliação de métodos para estimativa da evapotranspiração de referência no estado do Piauí. Revista da Sociedade Brasileira de Agrometeorologia, v. 11, n. 4, 10 ago. 2017.

LARMAN C. Utilizando UML e padrões: uma introdução à análise e ao projeto orientados a objetos e ao desenvolvimento iterativo. 3. ed. Porto Alegre: Bookman, 2007.

LEITÃO, M. DE M. V. B. R.; OLIVEIRA, G. M. DE; LEITÃO, T. J. V. Avaliação do desempenho de diferentes métodos de estimativa da evaporação para duas regiões da Paraíba. Revista Brasileira de Engenharia Agrícola e Ambiental, 2007.

LIU, Z.F. YANG, B.S. CUI. Spatial and temporal variability of annual precipittion during 1961-2006 in Yellow River Basin China. J. Hydrol., 361, pp. 330-338., 2008.

MAČEK, Urška; BEZAK, Nejc; ŠRAJ, Mojca. Reference evapotranspiration changes in Slovenia, Europe. Agricultural and Forest Meteorology, [s. 1.], v. 260-261, p. 183-192, 2018.

MCMAHON, T.A., PEEL, M.C., LOWE, L., SRIKANTHAN, R., MCVICAR, T.R.. Estimating actual, potential, reference crop and pan evaporation using standard meteorological data: a pragmatic synthesis. Hydrol. Earth Syst. Sc. 17 (4), 1331-1363, 2013.

MCVICAR, T.R., KÖRNER, C. On the use of elevation, altitude, and height in the ecological and climatological 
PEREIRA, D. dos R. et al. Desempenho de métodos de estimativa da evapotranspiração de referência para a região da Serra da Mantiqueira, MG. Ciência Rural, Santa Maria, v. 39, n. 9, p. 2488-2493, dez. 2009.

PILAU et al. Desempenho de métodos de estimativa da evapotranspiração de referência nas localidades de Frederico Westphalen e Palmeira das Missões, RS. Ciência Rural, Santa Maria, v. 42, n.2, p.283-290, fev. 2012.

PHP: Hypertext Preprocessor. 1995. Disponível em: http://php.net/.

PHP: What is PHP ?. Disponível em: https://secure. php.net/manual/pt_BR/intro-whatis.php. Acesso em: 03 de Fev. de 2019.

PHP: What can PHP do?. Disponível em: https://secure. php.net/manual/pt_BR/intro-whatcando.php. Acesso em: 03 de Fev. de 2019.

POSTGRESQL. What is PostgreSQL ?. Disponível em: https://www.postgresql.org/about/. Acesso em: 03 de Fev. de 2019.

ROSENBERRY, D.O., WINTER, T.C., BUSO, D.C.LIKENS, G.E. 2007, Comparison of 15 evaporation methods applied to a small mountain lake in the northeastern USA, Journal of Hydrology, vol. 340, no. 3, pp. 149-166.

SCHACH S. R. Engenharia de Software: Os Paradigmas Clássico e Orientado a Objetos. 7. ed. São Paulo: McGraw Hill, 2009.

SMARTY TEMPLATE ENGINE. Chapter 1. What is Smaty? Part I. Getting Started. Disponível em: https:// www.smarty.net/docs/en/what.is.smarty.tpl. Acesso em: 03 de Fev. de 2019.

SOMMERVILLE I. Engenharia de Software. 8. ed. São Paulo: Pearson Addison Wesley, 2007.

SOMMERVILLE I. Engenharia de Software. 9. ed. São Paulo: Pearson Addison Wesley, 2011.

SUlEIMAN, A. A.; HOOGENBOOM, G. Comparison of Priestley-Taylor and FAO-56 Penman-Monteith for daily reference evapotranspiration estimation in Georgia. Journal of Irrigation and Drainage Engineering, v.33, n.2, 175-182. 2007.

TABARI, H., GRISMER, M.E.TRAJKOVIC, S., Comparative analysis of 31 reference evapotranspiration methods under humid conditions, Irrigation Science, vol. 31, no. 2, pp. 107-117., 2013.

WANG, H. LIU, L. ZHANG, R. ZHANG. Climate change trend and its effects on reference evapotranspiration at Linhe Station, Hetao Irrigation District Water Sci. Eng., 7 (3) , pp. 250-266., 2014.
YANG, Q. LIU, B. Cui Spatial distribution and temporal variation of reference evapotranspiration during 19612006 in the Yellow River Basin China. Hydrol. Sci. J., 56 (6) , pp. 1015-1026, 2011.

\section{Contribuição dos autores}

Taison Anderson Bortolin

Contribuições substanciais para a concepção do trabalho; aquisição, análise e interpretação de dados; criação de novo software usado no trabalho; redigiu o trabalho e realizou uma revisão substancial.

Lucas Moraes dos Santos

Contribuições substanciais para a concepção do trabalho; análise e interpretação de dados; criação de novo software usado no trabalho; redigiu o trabalho e realizou uma revisão substancial.

Adriano Gomes da Silva

Contribuições substanciais para a concepção do trabalho; análise e interpretação de dados; criação de novo software usado no trabalho; redigiu o trabalho e realizou uma revisão substancial.

Vania Elisabete Schneider

Contribuições substanciais para a concepção do trabalho; redigiu o trabalho e realizou uma revisão substancial. 